\title{
Neonatal SARS-CoV-2 infection: is this a vertical transmission?
}

Femi Adeniyi ST6 Paediatrics, femi.adeniyi@nhs.net

Sanjeev Rath Neonatal Consultant, s.rath@nhs.net

Yewande Wey MTI Fellow, yewande.wey@nhs.net

Arrowe Park Hospital, Wirral University Teaching Hospital NHS Foundation Trust

Neonatal COVID-19, its manifestations and transmission, remains unclear. As the pandemic continues more evidence will emerge but so far, vertical transmission of COVID-19 is rare with just a few reports in the literature. ${ }^{1}$ We share our experience of managing a preterm newborn with COVID-19 in our neonatal intensive care unit (NICU) where the vertical route seems the most likely mode of transmission.

\section{The case}

A preterm female neonate was born at 31 weeks' gestation to a 26-year-old mother. The pregnancy was followed with routine antenatal scans and screening. The 28 -week scan showed a normal amniotic fluid volume and no concerns with lung development. There was no history of prolonged rupture of membrane. Three days prior to the delivery, the woman became unwell with fever, body aches and malaise and she presented to the hospital with tachycardia and low saturations. She needed oxygen through a face mask to maintain saturations. Her SARS-CoV-2 viral ribonucleic acid (RNA) reverse transcription polymerase chain reaction (RTPCR) test was positive.

The obstetric team decided to deliver the baby by emergency caesarean section due to reduced fetal movement, pathological cardiotocography and the unwell clinical state of the mother. Because of the urgency, there was not time to administer antenatal corticosteroids to accelerate fetal lung maturity; tocolysis was inappropriate. The neonatal team was present for delivery in full personal protective equipment (PPE) as per local guidelines.

A preterm infant was born in good condition but soon needed assistance with inflation and ventilation breaths due to a slow heart rate and poor respiratory drive. The newborn infant continued to need support with $50 \%$ oxygen at 10 minutes of age to maintain saturation within the acceptable range (>90\%). In view of prematurity and respiratory distress, the infant was transferred to the NICU's isolation room and commenced on continuous positive airway pressure (CPAP) ventilation. The preterm infant was intubated and received surfactant at one hour of age due to persistently increasing oxygen requirement and worsening respiratory acidosis. A chest X-ray (FIGURE 1) showed ground-glass opacification throughout the lungs. The infant required mechanical ventilation for 10 hours, non-invasive CPAP/high flow respiratory support for seven days, and further remained on low flow oxygen until day 10 of life. Sepsis investigations, as per National Institute for Health and Care Excellence and local guidance, were not suggestive of infection so antibiotics were stopped at 36 hours.

The newborn infant satisfied the British Association of Perinatal

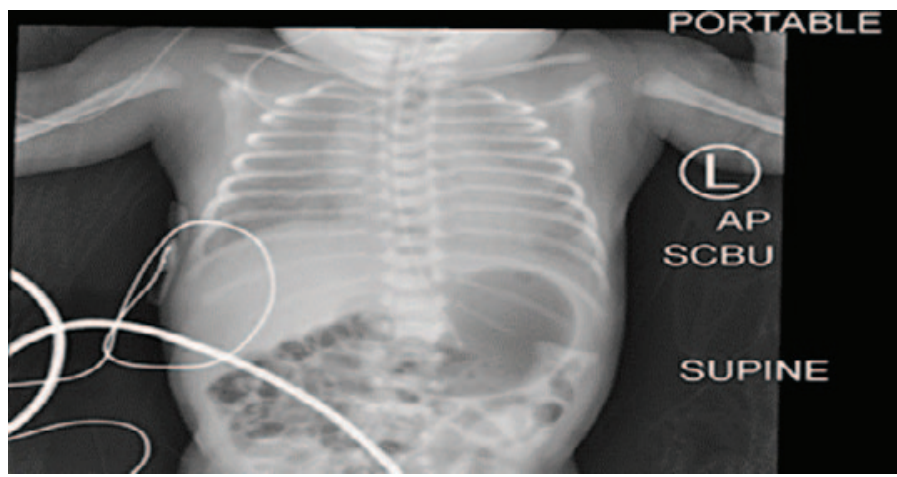

FIGURE 1 Chest X-ray showing ground-glass opacification throughout the lung.

Medicine's (BAPM) criteria for being managed as a suspected COVID-19 patient needing respiratory support. As part of screening, the infant had oropharyngeal swabs taken on days 3, 5, 7 and 10. The swabs tested positive for SARS-CoV-2 RNA by RTPCR on days 3, 7 and 10 .

The newly born baby was separated from the mother at birth. Neither parent could visit as the mother was in an isolation room on labour ward and the father had to isolate as being a contact trace. The parents had access to the baby through video calls until the 14 days of isolation passed, as per national and local policies. Throughout her care in the NICU, the infant remained in the isolation room in an incubator and staff wore full PPE during handling. The infant received screened donor breast milk during admission. The baby was discharged on day 14 of life and followup was arranged for six weeks later.

\section{Discussion}

The risk of preterm labour and fetal distress in mothers with severe viral infections such as influenza and SARS-CoV-2 is high., The incidence of preterm labour in COVID-19 positive pregnant women from the Wuhan meta-analysis report was $23 \%{ }^{4}$

Diagnosis of neonatal SARS-CoV-2 infection in a symptomatic exposed newborn is established with a positive nasopharyngeal swab for SARS-CoV-2 RNA by RT-PCR or by SARS-CoV-2 specific IgM antibody detection in the blood. ${ }^{5} \mathrm{~A}$ meta-analysis of 
74 reports identified 176 cases of neonatal SARS-CoV-2 infection where the authors demonstrated that vertical transmission occurred in $9 \%$ of the cases. One-third of the infections were acquired intrapartum; two-thirds acquired congenitally. To establish the timing of vertical transmission (in utero, intrapartum and/or early postnatal), it would be necessary to carefully evaluate the type and timing of samples. ${ }^{6}$ The presence of SARS-CoV-2 genome in vaginal swabs and breast milk raises the possibility of transmission to the baby either during birth (intrapartum route) or the early postnatal period. In addition, the presence of viral genome in umbilical cord blood increases the likelihood for placental transmission and makes in utero transmission plausible. ${ }^{7}$

\section{Neonatal COVID-19 or surfactant deficient lung disease?}

Our case was managed as a suspected case of COVID-19 as the infant was symptomatic with respiratory distress. Umbilical cord blood was not tested for SARS-CoV-2 but the infant had nasal and oropharyngeal swabs that were positive on days 3, 7 and 10 .

The clinical manifestation of respiratory distress needing invasive and non-invasive respiratory support along with the $\mathrm{X}$-ray findings, are commonly seen in preterm newborns with surfactant deficient lung disease (SDLD). Our team considered whether SARS-CoV-2 infection had any role to play here. The X-ray picture of typical COVID-19 in children shows bilateral peripheral, subpleural ground-glass opacities and consolidation. ${ }^{8}$ To our knowledge, there is not enough literature available to delineate the X-ray changes typical of SARS-CoV-2 pneumonia in newborn infants. A chest X-ray in one case report of neonatal COVID-19 demonstrated bilateral linear opacities. It also showed consolidation and pneumothorax in the right upper lobe. ${ }^{9} \mathrm{~A}$ second case report showed a ground-glass appearance on day 1 of life; this case went on to have a chest computed tomography scan on day 14 due to increased oxygen requirement and this showed lower lobe consolidation. ${ }^{10}$ Our case had a very similar groundglass appearance from day 1 but did not need further imaging as the condition improved by the 10th day.

\section{Conclusion and points for consideration}

We present a symptomatic case of an infant delivered by caesarean section, in isolation from the moment of birth, with the presence of SARS-CoV-2 RNA in multiple oropharyngeal swabs and a confirmed symptomatic SARS-CoV-2 infected mother. This leads us to postulate that vertical transmission is possible and is the most likely route here.

The clinical and radiological features of the preterm infant support a diagnosis of SDLD but raise the possibility that SARSCoV-2 contributed to the manifestations:

- did SARS-CoV-2 infection present as SDLD?

- did it make the manifestations of SDLD worse?

- was the SARS-CoV-2 infection merely coincidental?

\section{References}

1. Kotlyar AM, Grechukhina $\mathbf{O}$, Chen A, et al. Vertical transmission of coronavirus disease 2019: a systematic review and meta-analysis. Am J Obstet Gynecol 2021;224:35-53.e3

2. Meijer WJ, Van Noortwijk AG, Bruinse HW, Wensing AM. Influenza virus infection in pregnancy: a review. Acta Obstet Gynecol Scand 2015;94:797-819.

3. Nunes MC, Aqil AR, Omer SB, Madhi SA. The effects of influenza vaccination during pregnancy on birth outcomes: a systematic review and meta-analysis. Am J Perinatol 2016:33:1104-14

\section{The mother's perspective}

"On the day prior to delivery of my baby girl, I went to a GP hub with symptoms of COVID-19; they told me they were certain I had COVID but did not do a test because I had already sent a home test off. I remember sitting in the room at around $3 p m$ asking if COVID would affect my unborn daughter, to which they replied no, but we need to monitor her movements. I felt my baby moving, so I thought she was ok. The next morning, I woke up and realised I had not felt the baby move since $3 p m$ the previous day, so I phoned maternity triage. They told me to go and get checked, so I went at around 1.30pm that day.

I was put in a room because I had COVID; a nurse came in to monitor my baby's movements and heart beat. I heard my baby's heart beating away, so I thought everything was ok and I would be going home soon; about 20 minutes later the nurse came back in and did not say much. She had done a rapid COVID test which came back positive. I was nice and calm, expecting to be told everything was fine and that I could go home, but then a doctor/surgeon came in and said, we are taking you to the theatre now we need to get the baby out. My mind went blank I felt like I could not breathe. I was so confused I remember thinking why is this happening to me; is my baby ok? Then another two nurses came in and started taking my clothes off and trying to put stockings on me. The doctor shouted: there is no time for that, we need to go now.

I started to panic. My partner was not with me; I could not even tell him properly because they took me to theatre that fast. Luckily, my mum was outside and she could come in with me. It all happened so quickly and before I knew it, my baby was out. I got to have a quick look at her little face as she was whisked away from me. I do not remember much after that as I was poorly but when I was a bit better I asked if I could see my baby to which I was told I was not allowed because I had COVID and my baby was in isolation because she tested positive.

I will never forget the whole experience and it was the worst time of my life. I could not meet my baby for two weeks. I lost out on the magic hour - the special first moments - I only got to see my baby in pictures and on short videos. I was told COVID would not affect my baby, but it did. Now she is at home with her family and doing well. I am so grateful for everything the doctors and nurses did for my baby."

4. Gao Y-J, Ye L, Zhang J-S, et al. Clinical features and outcomes of pregnant women with COVID-19: a systematic review and meta-analysis. BMC Infect Dis 2020;20:564.

5. Raschetti R, Vivanti AJ, Vauloup-Fellous C, et al. Synthesis and systematic review of reported neonatal SARS-CoV-2 infections. Nat Commun 2020;11:5164.

6. World Health Organization. Definition and categorization of the timing of mother-to-child transmission of SARS-CoV-2. WHO; 8 Feb 2021 online at: www.who.int/publications/i/item/WHO-2019-nCoV-mother-to-childtransmission-2021.1

7. Fenizia C, Biasin M, Cetin I, et al. Analysis of SARS-CoV-2 vertical transmission during pregnancy. Nat Commun 2020;11:5128.

8. Foust AM, Phillips GS, Chu WC, et al. International expert consensus statement on chest imaging in pediatric COVID-19 patient management: imaging findings, imaging study reporting, and imaging study recommendations. Radiology Cardiothoracic Imaging 2020;2:e200214.

9. Coronado Munoz A, Nawaratne U, McMann D, et al. Late-onset neonatal sepsis in a patient with Covid-19. NEJM 2020;382:e49.

10. Abasse S, Essabar L, Costin T, et al. Neonatal COVID-19 pneumonia: report of the first case in a preterm neonate in Mayotte, an overseas department of France. Children 2020;7:87. 